\section{Twist in the tale}

\section{Clint McCrory}

A Topological Picturebook. By George K. Francis. Springer-Verlag: 1987. Pp.194. $D M 78, \$ 33$.

AFTER three decades of preoccupation with high dimensions, topologists have returned to the vivid world of surfaces and knots. An important factor contributing to the renaissance of visual topology has been the discovery by Bill Thurston of Princeton University of unexpectedly rich relations between non-Euclidean geometries and the topology of three dimensions.

But topologists are not very good at communicating their visions to others. Popular scientific writers still talk about turning coffee cups into doughnuts, leaving the fortunate impression that topologists merely belabour the visually obvious. What is needed is a modern version of the 1932 classic Anschauliche Geometrie, by Hilbert and Cohn-Vossen, with its emphasis on physical intuition rather than on abstract symbolism. George Francis's book is a step in the right direction. It is a manual on how to draw surfaces so that their topological complexity can be seen and understood.

Francis encourages the reader to learn to see by learning to draw. Many of his examples are entertaining visual brain teasers. Can you imagine a Moebius band whose border (edge) lies in a plane, or a two-sided surface whose border is a figureeight knot? The book can be enjoyed simply as a picture book: each of its 87 illustrations tells a story using a number of related figures, and understanding these little picture stories is a pleasant and often challenging exercise. The last three

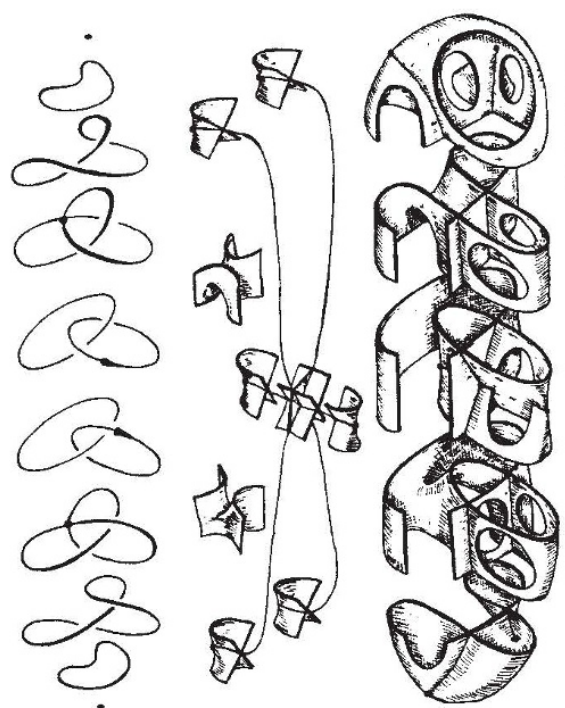

The Whitney bottle - recipe, middle, for the 3-dimensional shadow, right, of a Kleinbottle in 4-space from 3-dimensional sections.

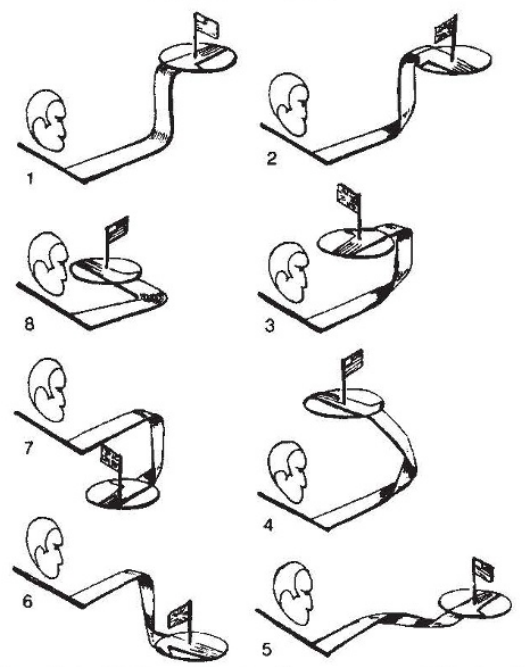

The plate trick - illustrating a geometrical principle popularized by Dirac, explained by Newman with the theory of braid groups.

chapters are much longer, and contain more intricate picture stories about important characters of modern topology: sphere eversions, mapping class groups and fibred knots (including some of Thurston's work). Through anecdotes, careful descriptions and references to

\section{Disorderly conduct}

Michael Berry

Chaos: Making a New Science. By James Gleick. Viking, New York: 1987. Pp.352. $\$ 19.95$.

IT Is now more than a decade since it began to be widely appreciated that systems may behave randomly in spite of being governed by causal laws whose mathematical formulation is simple. Since then, the concepts and methods of chaology have penetrated into virtually all branches of science. It is therefore surprising that we should have had to wait so long for a book-length elementary account of these developments, to complement the more philosophical and historical exposition by Prigogine and Stengers (Order Out of Chaos). And it is not a little shaming that the book should come from a journalist rather than one who is actively contributing to the subject.

The author has evidently taken a great deal of trouble to isolate the key ideas and explain them in simple terms. His technique is biographical: each concept is associated with one of the principal protagonists, whose character and personal history is interwoven with the science. The order thus imposed on the subject is not what would seem natural in a more didactic presentation - indeed the chapters could be arbitrarily permuted without losing clarity - but the immediacy and vividness provide some compensation. the literature, Francis skilfully unfolds the beauty of these subjects.

The foundation of the art of 'descriptive topology' is Hassler Whitney's theory of stable mappings of surfaces. The singularities (folds and cusps) of these mappings give visual clues to a surface's shape. (A corresponding theory of vision has been developed by the Dutch biological physicists Koenderink and van Doorn.) The topology of these singularities plays a fundamental role in René Thom's dynamical theory of catastrophes.

Computer graphics, the author contends, cannot yet compete with pen and paper in producing inexpensive, easily constructed images, and it is only in a postscript that Francis describes his initial forays into interactive animated computer graphics. I hope that he'll write a sequel on how to use a home computer to compose topological picture stories, for some of the most striking computer graphics of the past 15 years have been the result of mathematicians' attempts to see the objects they study.

Clint McCrory is a Professor in the Department of Mathematics, University of Georgia, Athens, Georgia 30602, USA.

Thus we are introduced to the mysteries of strange attractors through Edward Lorenz's early simulations of weather, Stephen Smale's mathematization of the stretching and folding of dough, David Ruelle's and Floris Takens's speculations on how fluids become turbulent, and the analogue computations of the 'Santa Cruz dynamical systems collective'.

Inevitably, the description of the central conception of fractals - geometric objects so wild that their dimensionality hovers between the integers - is enhanced by the larger-than-life personality of their creator, Benoit Mandelbrot. And the lonely, romantic genius of Mitchell Feigenbaum is pressed into service to emphasize his discovery that one of the ways in which chaos can develop is universal - that is, independent of the details of the system within wide limits.

Nobody should mistake this biographical approach for a serious history of the subject. It is a distortion to describe Laplace's determinism as "almost buffoonlike" just because that view has been superseded by the discovery of chaos. And the fact that Newton's theory of light eclipsed Goethe's theory of colour is no justification for calling the latter's obscurity "merciful".

Such inaccuracies are however unimportant by comparison with the unforgivable flaw in this book, namely its concentration on how the subject developed in North America, to the virtual exclusion of contributions by Soviet scientists. The seminal works of Kolmogorov 